\title{
Limitation of Intraoperative Transcranial Electrical Stimulation-Motor Evoked Potential Monitoring During Brain Tumor Resection Adjacent to the Primary Motor Cortex
}

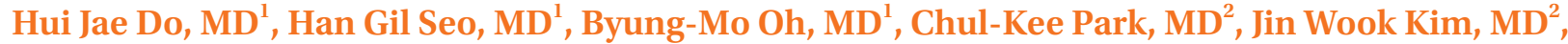 \\ Young Doo Choi, BS ${ }^{3}$, Seung Hak Lee, $\mathrm{MD}^{1}$
}

Departments of ${ }^{1}$ Rehabilitation Medicine and ${ }^{2}$ Neurosurgery, Seoul National University Hospital, Seoul National University College of Medicine, Seoul; ${ }^{3}$ Department of Neurosurgery, Seoul National University Hospital, Seoul, Korea

\begin{abstract}
Transcranial electrical stimulation-motor evoked potential (TES-MEP) is a valuable intraoperative monitoring technique during brain tumor surgery. However, TES can stimulate deep subcortical areas located far from the motor cortex. There is a concern about false-negative results from the use of TES-MEP during resection of those tumors adjacent to the primary motor cortex. Our study reports three cases of TES-MEP monitoring with falsenegative results due to deep axonal stimulation during brain tumor resection. Although no significant change in TES-MEP was observed during surgery, study subjects experienced muscle weakness after surgery. Deep axonal stimulation of TES could give false-negative results. Therefore, a combined method of TES-MEP and direct cortical stimulation-motor evoked potential (DCS-MEP) or direct subcortical stimulation should be considered to overcome the limitation of TES-MEP.
\end{abstract}

Keywords Transcranial electrical stimulation, Direct cortical stimulation, Intraoperative monitoring

\section{INTRODUCTION}

Transcranial electrical stimulation-motor evoked potential (TES-MEP) is a valuable intraoperative monitoring technique to monitor functional integrity of the cor- ticospinal tract during brain tumor resection [1]. In the area of intraoperative monitoring, the role of physiatrist is to predict motor outcomes and plan postoperative rehabilitation therapies based on its results. To predict motor outcomes, $50 \%$ amplitude reduction and $10 \%$ la-

Received February 7, 2018; Accepted May 10, 2018

Corresponding author: Seung Hak Lee

Department of Rehabilitation Medicine, Seoul National University Hospital, 101 Daehak-ro, Jongno-gu, Seoul 03080, Korea. Tel: +82-2-2072-3940, Fax: +82-2-743-7473, E-mail: seunghak@gmail.com

ORCID: Hui Jae Do (http://orcid.org/0000-0002-0041-9683); Han Gil Seo (http://orcid.org/0000-0001-6904-7542); Byung-Mo Oh (http://orcid. org/0000-0001-9353-7541); Chul-Kee Park (http://orcid.org/0000-0002-2350-9876); Jin Wook Kim (http://orcid.org/0000-0002-3773-3940); Young Doo Choi (http://orcid.org/0000-0002-1674-1095); Seung Hak Lee (http://orcid.org/0000-0002-3017-8497).

@ This is an open-access article distributed under the terms of the Creative Commons Attribution Non-Commercial License (http://creativecommons.org/ licenses/by-nc/4.0) which permits unrestricted noncommercial use, distribution, and reproduction in any medium, provided the original work is properly cited. Copyright $\odot 2018$ by Korean Academy of Rehabilitation Medicine 
tency prolongation of MEP are considered as significant deterioration criteria under TES-MEP monitoring [2]. Despite advantages of TES-MEP, deep axonal stimulation is a possible drawback. TES can stimulate deep subcortical areas such as the cerebral peduncle and pyramidal decussation [3]. This phenomenon has been demonstrated in several animal experiments and in electrical field modeling of TES $[3,4]$. There is a concealed concern for false-negative results caused by deep axonal stimulation during tumor resection around the primary motor cortex. However, there are only a few clinical mentions of this in the literature $[5,6]$.

On the other hand, direct cortical stimulation-motor evoked potential (DCS-MEP) has higher sensitivity and reliability than TES-MEP in insular and/or around precentral or postcentral neurosurgeries. Thus, DCS-MEP could be a more reliable method for avoiding deep axonal stimulation [7]. However, DCS-MEP during tumor resection around the primary motor cortex is not commonly used in Korea.

Our study reports three brain tumor cases of TES-MEP monitoring with false-negative results due to deep axo- nal stimulation during the resection of brain tumors in or adjacent to primary motor cortex. To avoid deep axonal stimulation of TES, we performed a combined method of TES-MEP and DCS-MEP and confirmed a true positive result.

\section{CASE REPORTS}

\section{Patient 1}

A 59-year-old woman presented with a history of seizure and muscle weakness in her right hand as grade 3 on the Medical Research Council (MRC) scale. Her FuglMeyer motor score at the right upper extremity was not measured. Brain magnetic resonance imaging (MRI) demonstrated a necrotic, hemorrhagic, peripheral enhancing lesion in the left precentral gyrus and area adjacent to the middle frontal gyrus (Fig. 1A). A craniotomy was performed for tumor removal under intraoperative TES-MEP monitoring. TES-MEP was evoked with repetitive stimulation of 6 pulses/train at 450 volts at C3/4 using an MEP monitoring system (NIM-Eclipse; Medtronic plc, Dublin, Ireland). Recording sites were bilateral del-
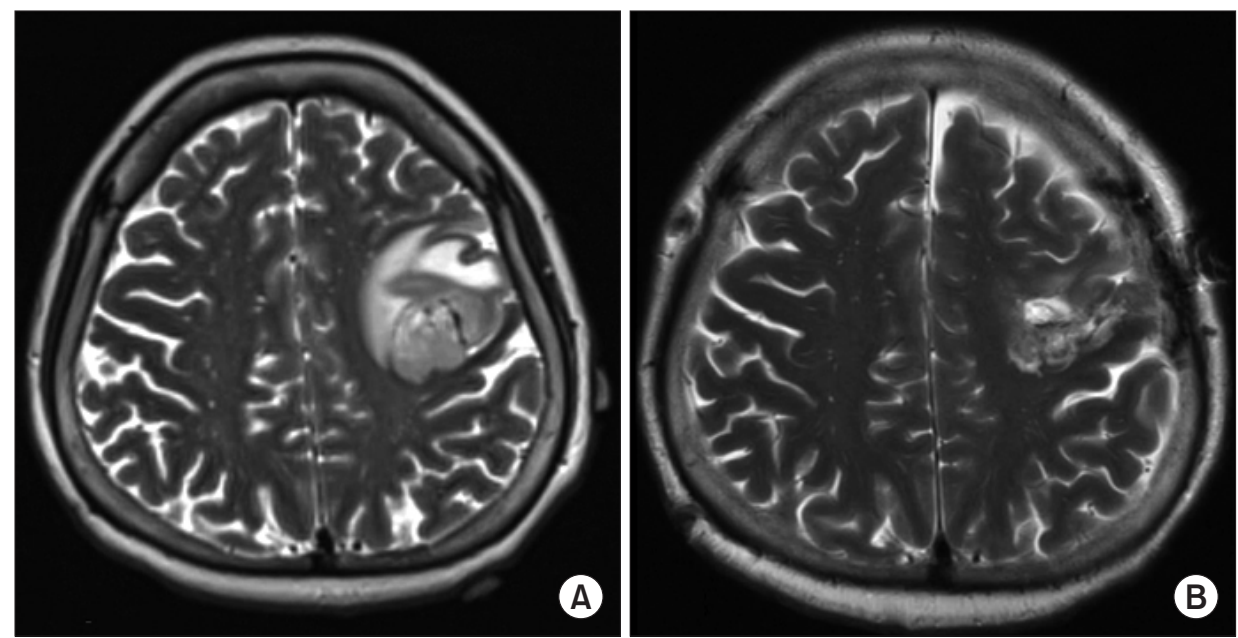

C

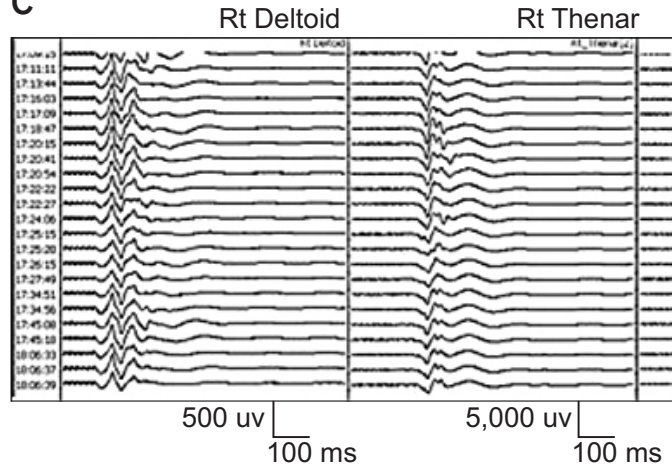

Rt Tibialis A.

Rt Abductor $\mathrm{H}$.

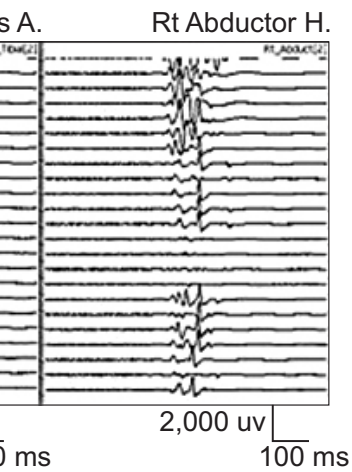

Fig. 1. Preoperative (A) and postoperative (B) axial T2-weighted magnetic resonance images of a lesion at the left precentral gyrus and area adjacent to the middle frontal gyrus which was removed with intraoperative transcranial electrical stimulation-motor evoked potential monitoring (see illustrative Patient 1). (C) Tracing shows transcranial electrical stimulation-motor evoked potential (TES-MEP) during the surgery. MEP amplitude was significantly decreased in the right lower extremity, but not in the right upper extremity. 
toid, abductor pollicis brevis, tibialis anterior, and abductor hallucis muscles. MEP amplitude was decreased significantly in the right lower extremity, but not in the right upper extremity (Fig. 1C). The amplitude of right median somatosensory evoked potential (SEP) was decreased. It did not recover at the end of surgery. Muscle weakness in both the right upper and lower extremities was aggravated after the operation to MRC scale 1. Fugl-Meyer motor score at the right upper extremity was 4 and that score at hand was 0 . Follow-up brain MRI demonstrated complete cortical tissue loss of the left precentral gyrus with white matter resection surface, suggesting possible acute infarction (Fig. 1B). Postoperative transcranial magnetic stimulation MEP was not performed. Upon discharge on postoperative day eight, her neurological status remained unchanged. Histology revealed glioblastoma (WHO grade IV).

\section{Patient 2}

A 54-year-old woman presented with a history of seizure. Brain MRI demonstrated a peripheral enhancing lesion at the left high frontal lobe involving the precentral gyrus (Fig. 2A). On admission, the patient was neurologically intact. Motor strengths of all extremities were measured as grade 5 on MRC scale. However, Fugl-Meyer motor score was not measured. A craniotomy was performed for tumor removal under intraoperative TES-MEP monitoring. TES-MEP was evoked with repetitive stimulation of 6 pulses/train at 350 volts at C3/4 using an MEP monitoring system (NIM-Eclipse). Recording sites were bilateral deltoid, abductor pollicis brevis, tibialis anterior, and abductor hallucis muscles. No significant change in TES-MEP or SEP was observed during the surgery (Fig. 2C). After the operation, neurological examination revealed motor weakness of the right upper extremity (MRC scale 2) as she was unable to grasp with her right hand. Fugl-Meyer motor score at the right upper extremity was 7 and that score at hand was 0 . Follow-up brain MRI demonstrated cortical tissue loss in the left precentral gyrus involving hand knob (Fig. 2B). Postoperative transcranial magnetic stimulation MEP elicited no response at the right abductor pollicis brevis muscle. At discharge
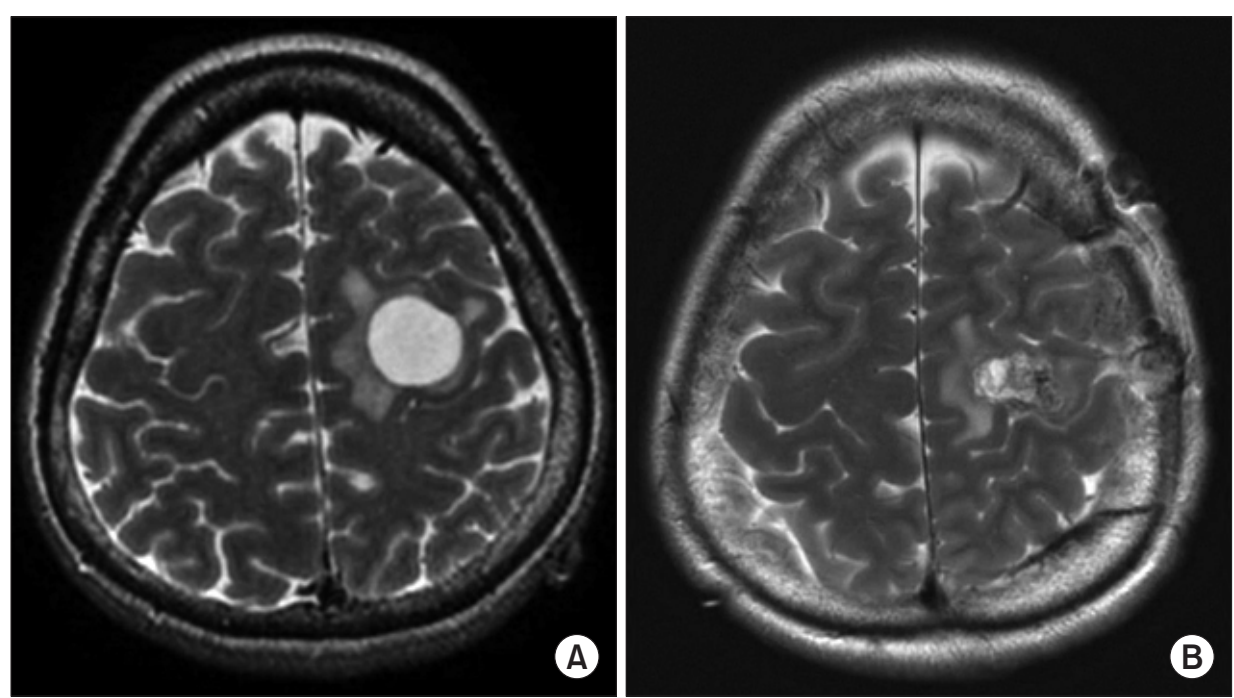

C

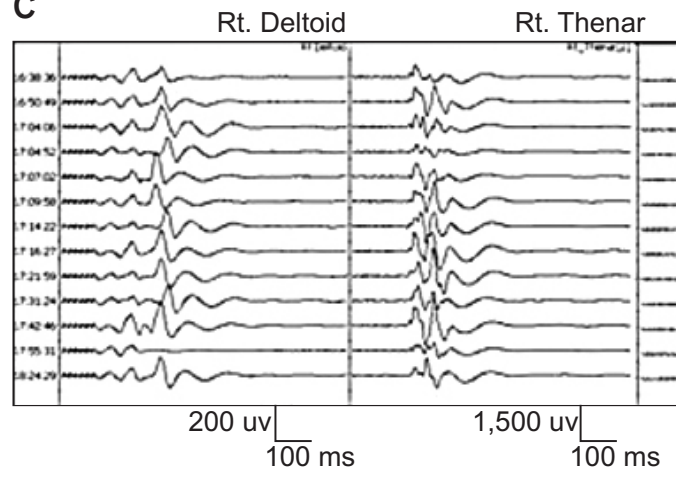

Rt. Tibialis A.

Rt. Abductor $\mathrm{H}$.

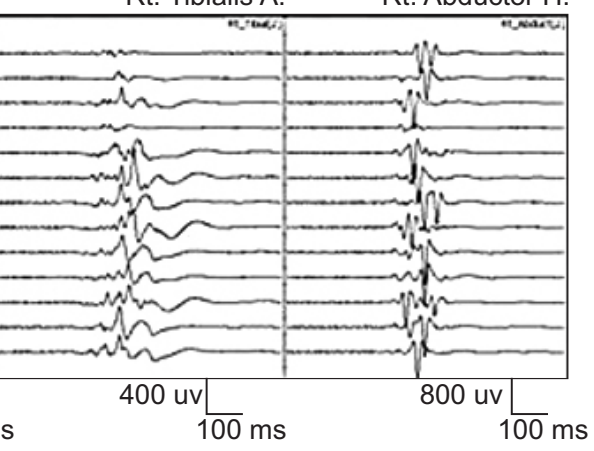

Fig. 2. Preoperative (A) and postoperative (B) axial T2-weighted magnetic resonance images of a lesion at the left high frontal lobe involving the precentral gyrus which was removed with intraoperative transcranial electrical stimulationmotor evoked potential monitoring (see illustrative Patient 2). (C) Tracing shows transcranial electrical stimulation-motor evoked potential (TES-MEP) during surgery. No significant change in TES-MEP was observed. 
on postoperative day nine, her neurological status was unchanged. Histology revealed pilocytic astrocytoma.

\section{Patient 3}

An 82-year-old woman presented with muscle weakness of the right upper extremity, predominantly in her hand (MRC scale 4). Her Fugl-Meyer motor score was not measured. Brain MRI demonstrated a mass in the left fronto-parietal lobe involving the precentral gyrus (Fig. 3A). A craniotomy was performed for tumor removal under intraoperative TES-MEP and DCS-MEP monitoring. TES-MEP was evoked with repetitive stimulation of 6 pulses/train at 450 volts at C3/4 while DCS-MEP was obtained with a strip electrode on the motor cortex through stimulation of 6 pulses/train at 300 volts using an MEP monitoring system (NIM-Eclipse). We used a 4-channel strip electrode in DCS which was inserted after craniotomy. The strip electrode was positioned at the motor cortex of precentral gyrus through SEP phase reversal, direct cortical stimulation, and correlation with navigation MRI. Recording sites were bilateral abductor pollicis brevis, abductor hallucis, right deltoid, and right tibialis anterior muscles. Because the tumor was located in the primary motor cortex, the surgeon and physiatrist for intraoperative monitoring predicted unavoidably significant signal loss in both TES-MEP and DCS-MEP during gross-total resection. Unexpectedly, no significant change in TESMEP at the right extremities was observed during surgery while significant loss in DCS-MEP was observed (Fig. 3C). The physiatrist discussed with the surgeon about an unexpected result and decided to perform repetitive TESMEP. Because the same result was observed in the repetitive stimulation, physicians concluded a false negative result from it and resection was continued. No significant change in SEP was observed during surgery. After the operation, neurological examination revealed motor weakness of the right upper and lower extremities (MRC scale 1). Her Fugl-Meyer motor score was not measured. Follow-up brain MRI demonstrated cortical tissue loss in the left fronto-parietal lobe involving the precentral gyrus (Fig. 3B). Postoperative transcranial magnetic stimulation MEP was not performed due to postoperative seizure. At discharge on postoperative day 13 , her neurological status was unchanged. Histology revealed glioblastoma.

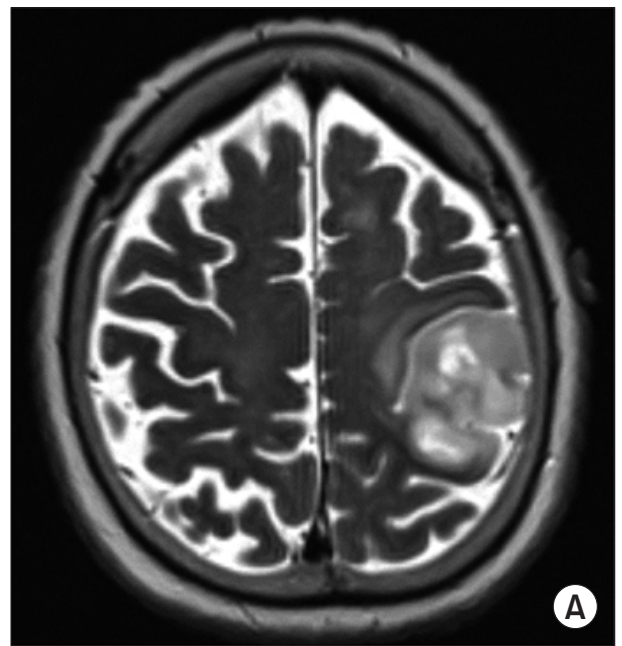

C

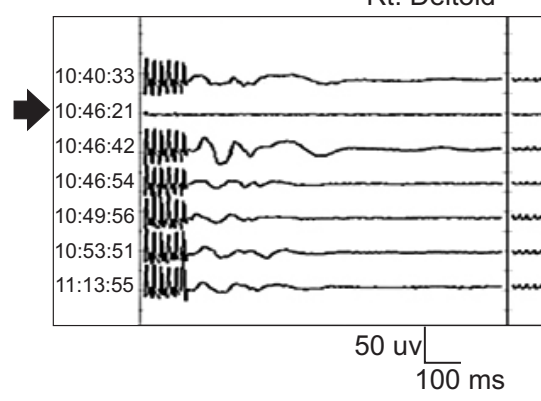

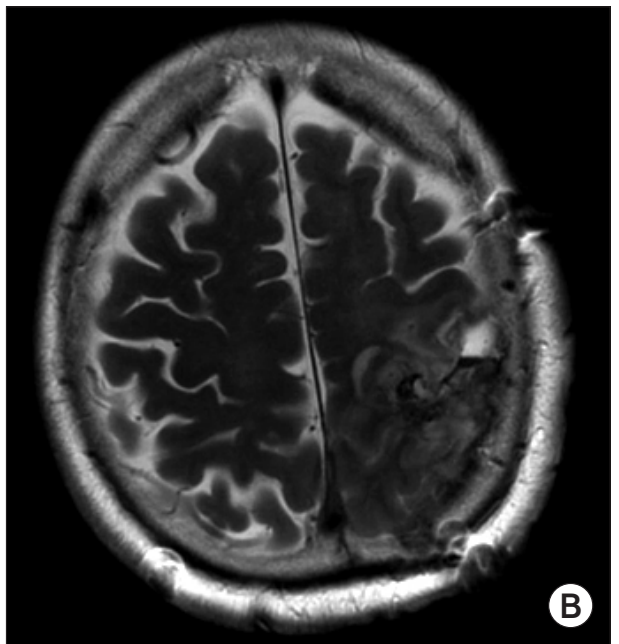

Rt. Thenar

Rt. Abductor $\mathrm{H}$.

Fig. 3. Preoperative (A) and postoperative (B) axial T2-weighted magnetic resonance images of a lesion at the left fronto-parietal lobe involving the precentral gyrus which was removed with intraoperative transcranial electrical stimulation and direct cortical stimulationmotor evoked potential monitoring (see illustrative Patient 3). (C) Tracing shows transcranial electrical stimulation-motor evoked potential (TES-MEP; waves other than arrow) and direct cortical stimulationmotor evoked potential (DCS-MEP; arrow) during surgery. While no significant change in TES-MEP of the right extremities was observed during surgery, significant loss in DCS-MEP was observed (arrow). 


\section{DISCUSSION}

Our study reported false-negative TES-MEP results in three cases of brain tumor resection due to deep axonal stimulation. When deep axonal stimulation occurs at subcortical areas, motor cortex injuries along the superficial subcortical motor pathways may go undetected, raising concerns for patient safety and reliability of TES-MEP monitoring.

There are several advantages of using TES-MEP under some clinical situations. TES-MEP can monitor the functional integrity of a wide corticospinal tract area. It may be better at detecting vascular compromises or remote area infarctions. In addition, TES-MEP can monitor possible delayed complications such as delayed ischemia because TES-MEP could be implemented until skin closure [8]. However, TES-MEP cannot detect superficial ischemia of the cortical branch or transient ischemia [8]. Conclusively, deep axonal stimulation can raise false negative results when using TES-MEP.

One possible way to overcome deep axonal stimulation of TES-MEP is to consider the advantage of DCS-MEP because DCS-MEP may detect injuries of pyramidal cells or their pyramidal axons promptly. With such advantage, DCS-MEP has higher sensitivity and reliability than TESMEP in insular and/or around precentral or postcentral neurosurgeries. Boex et al. [7] have reported the sensitivity of DCS-MEP for all new upper limbs deficits is $100 \%$, while the sensitivity of TES-MEP is only $82.3 \%$ in insular and/or around precentral or postcentral neurosurgeries. Therefore, DCS-MEP could be a more reliable method to avoid deep axonal stimulation. It is an alternative method of TES-MEP. Nevertheless, there are several disadvantages of DCS-MEP. It can evaluate only narrow area. In addition, insertion of strip electrodes at cortical areas of midline, sagittal plane, and subdural adhesions is difficult [8]. Moreover, there are unstable changes in DCS-MEP recordings such as abrupt fading of waveforms and intermittent changes in amplitude due to unknown causes [8]. Considering advantages and disadvantages of TES-MEP and DCS-MEP, a combined method of TES-MEP and DCS-MEP should be considered to monitor motor pathways effectively during resection of brain tumors located in or adjacent to the primary motor cortex. Even if there are discrepancies between results of TES-MEP and DCSMEP, results of DCS-MEP are weighted because of high sensitivities for these methods.

Another possible strategy is to use direct subcortical stimulation as an alternative or compensatory method. Direct subcortical stimulation mapping can stimulate white matter bundles and protect deep subcortical corticospinal tract pathways in white matter around resection area. Keles et al. [9] have reported that the surgeon could achieve an acceptable risk of permanent motor deficits in resection of gliomas located within or adjacent to the descending motor pathways. For that reason, a combined method of DCS-MEP and subcortical stimulation is the gold standard to preserve structures of the primary motor cortex and pyramidal tract [10].

There is a limitation in these cases. There were no longterm follow-ups of neurological status in these patients. The long-term follow-up is needed to exclude the possibility of supplementary motor area syndrome and recovery of motor function by reducing brain edema.

In conclusion, deep axonal stimulation of TES during brain tumor resection in or adjacent to the primary motor cortex could give false-negative results in intraoperative monitoring as a limitation of TES. Therefore, a combined method of TES-MEP and DCS-MEP or direct subcortical stimulation should be considered to overcome the limitation of TES-MEP.

\section{CONFLICT OF INTEREST}

No potential conflict of interest relevant to this article was reported.

\section{REFERENCES}

1. Zhou HH, Kelly PJ. Transcranial electrical motor evoked potential monitoring for brain tumor resection. Neurosurgery 2001;48:1075-81.

2. Neuloh G, Pechstein U, Cedzich C, Schramm J. Motor evoked potential monitoring with supratentorial surgery. Neurosurgery 2004;54:1061-72.

3. Rothwell J, Burke D, Hicks R, Stephen J, Woodforth I, Crawford M. Transcranial electrical stimulation of the motor cortex in man: further evidence for the site of activation. J Physiol 1994;481(Pt 1):243-50.

4. Holdefer RN, Sadleir R, Russell MJ. Predicted current densities in the brain during transcranial electrical stimulation. Clin Neurophysiol 2006;117:1388-97. 
5. Szelenyi A, Kothbauer K, de Camargo AB, Langer D, Flamm ES, Deletis V. Motor evoked potential monitoring during cerebral aneurysm surgery: technical aspects and comparison of transcranial and direct cortical stimulation. Neurosurgery 2005;57(4 Suppl):331-8.

6. Macdonald DB. Intraoperative motor evoked potential monitoring: overview and update. J Clin Monit Comput 2006;20:347-77.

7. Boex C, Haemmerli J, Momjian S, Schaller K. Prognostic values of motor evoked potentials in insular, precental, or postcentral resections. J Clin Neurophysiol 2016;33:51-9.

8. Motoyama Y, Kawaguchi M, Yamada S, Nakagawa I, Nishimura F, Hironaka Y, et al. Evaluation of combined use of transcranial and direct cortical motor evoked potential monitoring during unruptured aneurysm surgery. Neurol Med Chir (Tokyo) 2011;51:1522.

9. Keles GE, Lundin DA, Lamborn KR, Chang EF, Ojemann G, Berger MS. Intraoperative subcortical stimulation mapping for hemispherical perirolandic gliomas located within or adjacent to the descending motor pathways: evaluation of morbidity and assessment of functional outcome in 294 patients. J Neurosurg 2004;100:369-75.

10. Saito T, Muragaki Y, Maruyama T, Tamura M, Nitta M, Okada Y. Intraoperative functional mapping and monitoring during glioma surgery. Neurol Med Chir (Tokyo) 2015;55:1-13. 